\title{
DA JAMES JOYCE A JOHN ASSURBANIPAL SMITH. LA (DE)LEGITTIMAZIONE DEL COMICO NELL'OPERA DI UMBERTO ECO
}

\section{GIOVANNI TISO}

\begin{abstract}
Sommario
This essay explores the role of humour and the treatment of the comic in the works of Umberto Eco, and in charting their evolution - from the earnest beginnings of Opera Aperta to the confident use of wit and parody in the more mature works - it seeks to highlight a contradiction: even as on the one hand he is willing to regard the comic as a legitimate object of critical attention, and gives it a central thematic role in The Name of the Rose, Eco stops short of fully acknowledging its critical dimension and its potential to subvert traditional cultural hierarchies and roles.
\end{abstract}

Grande tra gli uomini e di gran terrore è la potenza del riso: contro il quale nessuno nella sua coscienza trova sé munito da ogni parte. Chi ha coraggio di ridere, è padrone del mondo, poco altrimenti di chi è preparato a morire. Giacomo Leopardi, Pensieri LXXVII

"Avrei voluto comporre un'opera filosofica fatta solamente di scherzi." "Perché non l'hai fatto?" "Mi mancava il senso dell'umorismo." Wittgenstein, di Derek Jarman

Tra le varie disquisizioni teologiche e filosofiche che attraversano $\mathrm{Il}$ nome della rosa, ce n'è una che acquista via via un ruolo chiave: è la disputa sulla "liceità del riso" (Il nome della rosa, 1980:137) tra Guglielmo e Jorge e che fa da sfondo alla catena di omicidi che si svolgono intorno all'abbazia. Di per sé la questione sembrerebbe 
anacronistica, strettamente confinata all'epoca in cui è ambientato il romanzo: se sia consentito o meno fare battute di spirito, o esprimersi in maniera irriverente su argomenti come politica o religione, è un problema che ovviamente ai nostri tempi non si pone, o per lo meno che non si pone nei termini nei quali lo intende Jorge da Burgos. Prima ancora che questo personaggio entri fisicamente in scena, a precederlo è un ammonimento tratto dalla regola benedettina: "Verba vana aut risui apta non loqui" (86). Le parole vane e che si prestano al riso sono per Jorge sinonimo di un "discorso cattivo" (87), da cui il monaco deve astenersi (anche per distinguersi dai francescani come Guglielmo, che Jorge accusa di trattare con indulgenza "anche la giocondità più inopportuna" (87). In seguito questo invito alla serietà e al decoro si rivelerà un'ossessione dai risvolti omicidi: Jorge è talmente terrorizzato dallidea che altri possano impadronirsi dell'unica copia superstite del secondo libro della Poetica di Aristotele, di cui è custode, da cospargerme di veleno le pagine, in modo che coloro che oseranno leggerlo ne restino uccisi.

Dicevo che la questione ai tempi nostri difficilmente si porrebbe in questi termini, e in effetti sarebbe un po' difficile immaginare una catena di delitti con un movente simile ambientati, chessò, nel Piemonte del tardo Novecento. Tuttavia la cornice del romanzo invita esplicitamente il lettore, seppure con formula ironica, a fare un parallelo con l'Italia contemporanea:

Negli anni in cui scoprivo il testo dell'abate Vallet circolava la persuasione che si dovesse scrivere solo impegnandosi sul presente, e per cambiare il mondo. A dieci e più anni di distanza è ora consolazione dell'uomo di lettere (restituito alla sua altissima dignità) che si possa scrivere per puro amore di scrittura. $\mathrm{E}$ così ora mi sento libero di raccontare, per semplice gusto fabulatorio, la storia di Adso da Melk, e provo conforto e consolazione nel ritrovarla così incommensurabilmente lontana nel tempo, [...] così gloriosamente priva di rapporto coi tempi nostri [...]. (15) 
Questa cornice, di per sé comica per come costruisce la finzione del manoscritto ritrovato da un Eco anche lui fittizio (qui nella veste di intellettuale disimpegnato e sussiegoso filologo), invita a leggere il romanzo come una chiave per discutere argomenti cari al 'vero' Eco. Vediamo allora se ha senso fare il seguente parallelo: che tra gli intellettuali contemporanei di Eco ve ne fossero di due tipi, quelli severi e mortalmente seri, alla Jorge da Burgos, impegnati nella difesa della cultura 'alta'; e quelli bonari e arguti, alla Guglielmo da Baskerville, in grado di parlare la lingua dei colti ma anche di difendere il comico in quanto lingua degli incolti, ovvero della massa. I primi sarebbero gli apocalittici, i custodi della sacralità del sapere dei pochi; mentre i secondi non sarebbero gli integrati, bensì gli intermedi, i personaggi alla Eco, capaci di tenere il piede in due scarpe, ovvero di abitare la cittadella del sapere dei pochi ma anche di uscirne talvolta per ridere dal di fuori della serietà e seriosità dei suoi abitanti. Che i primi fossero la maggioranza, e che l'accademia italiana si fosse data una regola sul tipo di quella dei benedettini ce lo dice un altro illustre professore, Guido Almansi (1986), quando scrive:

[...] il colossale processo ipocrita di sublimazione che si chiama cultura continua a privilegiare il serio sul suo contrario (che i nemici chiamano faceto), il tragico sul comico, la mutria sul sorriso, le ciglia aggrottate sull'ironia. Errore gigantesco che ha da sempre traviato la nostra visione di ciò che è cultura, cioè di ciò che è precipuamente umano nell'uomo. (9)

Vedremo come Eco sembri a prima vista allineato con queste posizioni, ma anche come il suo discorso sul comico non sia privo di contraddizioni e non costituisca fino in fondo una legittimazione.

Per prima cosa bisognerebbe fermarsi e dare una definizione di cosa sia il comico, ma rinuncio subito. In questo mi sento confortato da tutti quelli che hanno gettato la spugna prima di me, compreso lo 
stesso Eco, che nel saggio "Ma che cosa è questo Campanile" (1985) scrive che a voler definire tutti i meccanismi del comico senza essere preparati "si rischia di diventare un personaggio di Campanile" (Sugli specchi e altri saggi, 1985:271). Tipico di queste definizioni è lo sforzo di esaurire la vastissima gamma di fenomeni che rientrano nel senso comune del termine, finendo inevitabilmente con l'essere troppo riduttivi o troppo vaghi. Ma dal momento che tutti questi tentativi si basano pur sempre sull'esistenza di un senso comune del significato del termine, tanto vale usare quello e limitarsi a dire che per comico s'intende ciò che s'intende per comico. Del resto la viscosità di categorie quali comico, umorismo, ironia, satira, parodia, ecc. è tipica anche delle definizioni abbozzate dallo stesso Eco, di solito invece molto rigoroso nelle distinzioni terminologiche. Così la spiegazione di cosa sia la risata nel Trattato di semiotica generale (1975): "La risata costituisce [...] l'effetto collaterale di un uso improprio del codice o della scoperta di una contraddizione interna al codice" (95) - si ritrova nella versione inglese, curata dallo stesso Eco, ma riferita questa volta al comico (A Theory of Semiotics, 1976:64). Per non parlare del fatto che il Trattato non chiarisce perché certi usi impropri del codice facciano ridere e altri no.

In questo saggio userò allora il "comico" come termine-ombrello da applicare nella sua accezione più ampia per evidenziare determinati aspetti dell'opera di Eco. I suoi primi due libri si prestano subito a questa non-definizione, giacché alla micidiale serietà dell'Opera aperta (1963) si contrappone la varia comicità del Diario minimo (1992). Quello che colpisce anzi di questi due testi è proprio quanto sia serio l'uno e quanto sia divertente l'altro. Il rapporto tra le due opere prime, cui vanno aggiunti i saggi de Le poetiche di Joyce in quanto originariamente inclusi in Opera aperta, è indicativo insomma dell'atteggiamento ancora ambivalente di Eco nei confronti del comico. In Opera aperta esso manca in maniera pressoché totale, e si tratta di un'assenza tanto più eclatante se si fa il paragone con i saggi successivi. Una volta sola in questi primi saggi 'seri' il giovane Eco si lascia andare a una battuta. Lo fa per mettere in ridicolo le tesi di 
coloro che criticarono Robbe-Grillet per avere descritto un incidente capitatogli secondo i canoni della narrativa tradizionale, anziché alla maniera del nouveau roman: "Nessuno pretenderebbe," ribatte Eco, "che uno studioso di geometrie non-euclidee, dovendo misurare la propria stanza per costruire un armadio, usasse la geometria di Riemann [...]" (Opera aperta, 1963:207). Per il resto la prosa di Opera aperta è talmente seria da sconfinare spesso nel serioso, e non sono rari i passaggi come il seguente:

In un quadro di Pollock non ci viene rappresentato un universo figurativo conchiuso: l'ambiguo, il vischioso, l'asimmetrico vi intervengono proprio per far sì che lo spunto plastico-colorifero prolifichi continuamente in una incoattività di forme possibili. (218)

Il Diario minimo, la rubrica curata da Eco in quegli anni sul Verri, gli dava la possibilità di distrarsi dalle incoattività di forme possibili. La raccolta pubblicata da Bompiani nel 1963 con lo stesso titolo della rubrica, e giunta fino a noi attraverso una serie di revisioni (di cui l'ultima nel 1975) contiene a detta dell'autore "quasi esclusivamente pastiches e parodie" (Diario minimo, 1992:7). Nell'introduzione Eco spiega: "Quando scrivevo questi pezzi lo facevo con un poco di imbarazzo, come se non fosse decoroso scherzare su pratiche culturali che io stesso in altra sede seguivo" (8). Ma con il passare degli anni subentrò in lui il conforto della convinzione che "una delle prime e più nobili funzioni delle cose poco serie è di gettare un'ombra di diffidenza sulle cose troppo serie - e tale è la funzione seria della parodia" (8).

L'introduzione a Diario minimo è prima di tutto una giustificazione del perché i pezzi siano stati pubblicati, casomai si pensasse che sono il frutto di attività fini a se stesse (e pertanto, implicitamente, non legittime). Nel darla, Eco sceglie di concentrarsi solo sulla parodia e per giunta ne ammorbidisce la portata critica, spiegando che "non sempre una parodia si esercita su un modello che 
considera negativo; sovente parodiare un testo significa anche rendergli omaggio" (8). Se l'assoluta serietà di Opera aperta segnala la ritrosia, anche comprensibile, dell'Eco esordiente nei confronti di certe strategie retoriche in ambito accademico, questa introduzione ci rivela che quella ritrosia non era del tutto scomparsa quindici anni e molti saggi dopo. Perché ridurre la raccolta, tra l'altro di grande successo, alla sola parodia? Fanno eccezione infatti almeno tre testi, e tra questi (insieme a "Elogio di Franti" e "Lettera a mio figlio") c'è anche il più rappresentativo, cioè la "Fenomenologia di Mike Buongiorno," che è satira e pure piuttosto feroce:

Mike Bongiorno non si vergogna di essere ignorante e non prova il bisogno di istruirsi. Entra a contatto con le più vertiginose zone dello scibile e ne esce vergine $\mathrm{e}$ intatto, confortando le altrui tendenze all'apatia e alla pigrizia mentale. Pone gran cura nel non impressionare lo spettatore, non solo mostrandosi all'oscuro dei fatti, ma altresì decisamente intenzionato a non apprendere nulla. (31)

Neanche l'Elogio di Franti è una parodia, bensì un vero e proprio discorso sul comico. Preceduto da una citazione dal saggio sull'umorismo di Baudelaire - paragrafo che si conclude con la frase "il riso è satanico: è dunque profondamente umano" (81) - il pezzo offre una rivalutazione del personaggio deamicisiano di Franti e della sua risata, diabolica e come tale appunto profondamente umana. Partendo da una sana decostruzione del personaggio narrante di Enrico - educato "alla violenza e alla retorica nazionale, all'interclassismo corporativista, e all'umanitarismo paternalista" (83) - Eco loda Franti per la sua capacità di sfuggire al sentimentalismo del libro e del suo autore, e ne fa un personaggio addirittura rivoluzionario proprio in virtù della sua famosa, 'infame' risata:

Ecco dunque profilarsi l'idea di un Franti come motivo metafisico nella sociologia fasulla del Cuore. Il riso di 
Franti è qualcosa che distrugge, ed è considerato malvagità solo perché Enrico identifica il Bene all'ordine esistente e in cui si ingrassa. Ma se il Bene è solo ciò che una società riconosce come favorevole, il Male sarà soltanto ciò che si oppone a quanto una società identifica con il Bene, ed il Riso, lo strumento con cui il novatore occulto mette in dubbio ciò che una società considera come Bene, apparirà col volto del Male [...]. (89)

Il comico emerge qui come strumento talmente potente da sovvertire i significati voluti dall'autore stesso (nel senso di De Amicis).

Ben più in là dei sentimenti espressi nell'introduzione vanno anche i due versi di Joyce scelti per l'epigrafe: "music-hall not poetry/ is a criticism of life". Eccoci in pieno nel terreno della legittimazione, dove il comico viene investito di una dignità uguale se non superiore addirittura alla poesia. A maggior ragione stupisce allora che nel dedicare un intero libro allo studio dello stesso Joyce, Eco non dedichi neanche un paragrafo al ruolo del comico nelle sue opere. Discutendo sì l'uso del calembour in Finnegans Wake, ma solo per la sua capacità di farsi "immagine delle relazioni possibili tra gli eventi dell'universo" (Le poetiche di Joyce, 1962:127) e non per la sua perfino ovvia dimensione comica ${ }^{1}$.

Con il passare degli anni certe ritrosie in parte rimangono, come vedremo, ma viene decisamente superata la netta separazione tra l'Eco saggista e l'Eco umorista. Oltre ad ampliare e rivedere periodicamente Diario minimo, Eco inizia a trasferire nei saggi la prosa brillante di testi come la Fenomenologia. Il comico diventa così strategia retorica in grado di potenziare l'efficacia del messaggio, a prescindere dal contenuto.

Joyce stesso era solito lamentarsi del fatto che la critica non parlasse mai di Ulysses e Finnegans Wake come testi comici. Si veda ad esempio il James Joyce di Richard Ellman: "Joyce commented to Georg Goyert of Jung, 'He seems to have read Ulysses from first to last without one smile. The only thing to do in such a case is to change one's drink"' (1959: 641). 
Il Trattato di semiotica generale è l'esempio più eclatante. Il progetto non è meno ambizioso di Opera aperta - si tratta nientemeno che di "delineare una teoria globale di tutti i sistemi di significazione e i processi di comunicazione" (Trattato di semiotica, quarta di copertina) - ma qui Eco si concede qualche momento di autoironia. Spiega ad esempio di aver scelto un titolo che fa il verso ad Einstein solo dopo aver scartato - per "timore reverenziale, senso della misura e senso dell'umorismo" - un non meno impegnativo Critica della semiotica pura e della semiotica pratica, e che del resto questo suo lavoro, rispetto a quello dell'illustre filosofo tedesco, ha un vantaggio: "è decisamente più noioso" (6). Il Trattato ricorre poi a artifici comici per variare il registro e mantenere desta l'attenzione del lettore. È il caso della scelta in certi esempi di nomi improbabili come "John Assurbanipal Smith" (126), o di vocaboli fumettistici come "dimetilcaciocavalpirazolone" (127), o di frasi che attentano alla serenità del lettore come "Il tuo gatto sta annegando nella pentola del minestrone" (96). A Eco piace pure divertire se stesso: ecco allora che il corrispettivo nella versione inglese della frase "Giulio Cesare morì nel 44 a.C." (96) è un inopinato "Napoleon died at Saint Helena on May 5, 1821" (A Theory of Semiotics, 1976:65), laddove lo scambio degli imperatori non si spiega se non con il gusto di Eco per il gioco, anche quando esso non coinvolge il lettore e come tale risulta effettivamente 'fine a se stesso'.

C'è affinità tra il gioco e il comico, nella misura in cui entrambi sono imparentati con il divertimento, ed è nella loro intersezione che si collocano una serie di opere di Eco non altrimenti definibili. È il caso della traduzione degli Esercizi di Stile (1983) di Raymond Queneau, raccolta di novantanove variazioni sul tema di un banalissimo racconto il cui fascino consiste nella capacità dell'autore di giocare con generi letterari, figure retoriche, permutazioni lessicali e sintattiche, con risultati spesso oggettivamente divertentissimi. L'estrema difficoltà della traduzione fa della versione italiana di Eco un testo per certi versi del tutto nuovo, che dell'originale conserva lo spirito e le regole, ma che può essergli fedele solo concedendosi non 
poche sostanziali libertà. "Fedeltà", scrive Eco nell'introduzione, "significava capire le regole del gioco, rispettarle, e poi giocare una nuova partita con lo stesso numero di mosse" (Queneau, 1983:xix).

È in esercizi come questi che l'umorismo di Eco si esprime al meglio. Così il gioco dei titoli cinematografici, creato insieme a Roberto Benigni e approdato sulla rivista on line Golem (co-fondata dallo stesso Eco) prima di essere trasformato a sua volta in un libro', ricorda da vicino la Facoltà di Irrilevanza Comparata creata da Belbo e Diotallevi ne Il pendolo di Foucault (1988), un passatempo per persone colte (per poter collocare l'Ippica Azteca nel dipartimento bisogna innanzitutto sapere che gli aztechi non conoscevano il cavallo) e al solo scopo di divertirsi. Il Secondo diario minimo (1992) rientra più del primo in questo filone del gioco letterario e linguistico.

Un altro aspetto di questi esercizi è la loro capacità di far comprendere meglio i meccanismi del linguaggio. Eco ce lo ricorda nell'introduzione a Esercizi di stile, che fu adottato da molte scuole e che ha ispirato tentativi di emulazione come I draghi locopei ${ }^{3}$ dell'insegnante Ersilia Zamponi, come pure in Povero Pinocchio (1995), raccolta degli esercizi degli studenti di un seminario di composizione tenuto da Eco al Dams di Bologna.

Alla passione di Eco per il gioco appartiene anche un saggio sull'Homo Ludens di Huizinga, nella quale si sottolinea tra l'altro come la rivalutazione del gioco implichi la messa in discussione delle gerarchie culturali:

2 Cfr Bartezzaghi (1998).

3 Zamponi (1986). Nella presentazione del volume, Eco rilancia l'importanza degli esercizi di stile e i loro tratti in comune con le metafore poetiche: "La scuola come gioco, piacere, divertimento. In cui non solo si impara, ma si fa quello che gli scrittori di tutti i tempi hanno fatto, si capiscono le potenze bifide, esplosive nel linguaggio; e col linguaggio si esplorano i meandri della coscienza. Alle origini, enigma, poesia e metafora sono strettamente intrecciati, Aristotele lo sapeva. La più alta delle metafore poetiche e il più meccanico degli enigmi hanno in comune il fatto che le parole possono dire più di quello che sembrano dire. Tra gioco di parole, lapsus, sogno e invenzione corrono legami sottili." (VII). 
[...] la nozione di gioco come costante dei comportamenti culturali affascinava se non altro perché era oltraggiosa aveva tutta l'aria di uno pseudoconcetto che prendeva violentemente il potere insediandosi nel palazzo d'Inverno sino ad allora alteramente abitato dall'Estetica, dalla Teoretica, dall'Etica e dall'Economia (Sugli specchi, 1985:284).

Per traslato, legittimare il comico vorrà dire allora non solo praticarlo a margine di attività più serie, o prenderne a prestito certe forme per un uso retorico in altri contesti, ma farne vero e proprio oggetto di analisi culturale. È precisamente ciò che Eco fa a partire da Apocalittici e integrati (1965), dove la nozione di cosa sia un prodotto culturale 'minore' viene messa in discussione applicando l'intero armamentario degli strumenti critici allo studio di opere tradizionalmente trascurate dai critici.

Passiamo allora agli scritti di Eco sul comico, quelli in cui dovrebbe emergere in maniera più esplicita il tema della sua legittimazione. Oggetto di questa breve analisi saranno il capitolo sul fumetto di Charles Schulz in Apocalittici e integrati, un saggio su Pirandello dal titolo "Pirandello ridens" (1967), l'introduzione a Se la luna mi porta fortuna di Campanile (1975) e il contributo di Eco ad uno studio sul fenomeno del carnevale ("The Frames of Comic 'Freedom"', 1984).

Questi testi indicano prima di tutto la disponibilità di Eco a riconoscere la grandezza in senso assoluto di alcuni autori comici. In particolare Schulz e Campanile vengono lodati in termini pressoché identici, cioè per la loro capacità di illuminare certi aspetti della condizione umana ${ }^{4}$. Altro aspetto ricorrente come detto e che il

4 Si tratta dei paragrafi conclusivi dei due saggi: "Qui [1'umorismo di Campanile] è chiave per capire, attraverso le contorsioni del linguaggio comune, restituito alla sua pregnanza dalla tecnica amplificatoria, un aspetto sia pure minimo della condizione umana." ("Ma che cosa è questo Campanile', Sugli specchi 1985:279). E anche: "Schulz continuerà a mostrarci nel volto di Charlie Brown, con due colpi di matita, la sua versione della condizione umana" ('Il mondo di Charlie Brown', Apocalittici e integrati 1965:276). 
comico non viene trattato da Eco come un campo ben definito o chiaramente definibile, trattandosi di "un'esperienza molto imprecisa, tanto è vero che essa va sotto nomi diversi, quali Comico, Umorismo, Ironia, ecc." (Sugli specchi, 1985:261). L'unica distinzione terminologica che si concede Eco è però di una certa importanza, e riguarda la differenza tra comicità e umorismo, osservata in Pirandello e ripresa nel testo sul carnevale. Il comico, secondo Pirandello, nascerebbe da un venir meno delle attese che ci lascia "piacevolmente e diabolicamente sorpresi" (Sugli specchi, 264), e che si accompagna al "senso di superiorità" (265) che deriva dall'essersi accorti di un errore altrui. L'umorismo si coincide invece con la rinuncia al distacco e alla superiorità, ovvero con la riflessione "che si esercita prima o dopo il comico" (265).

Il comico in questa prospettiva è davvero diabolico, come lo voleva Baudelaire, ma in compenso ha perso la possibilità di riscatto sociale avanzata ne L'elogio di Franti. Anzi, nel saggio sul carnevale questa dimensione viene addirittura ribaltata. In contrapposizione alle tesi espresse da Bakhtin nel suo studio dell'opera di Rabelais, Eco afferma qui che il comico carnascialesco (la cui versione contemporanea sarebbe il varietà massmediale) non è altro che una trasgressione tollerata dal sistema e asservita ad esso ("The Frames of Comic Freedom", 1984:7). La conclusione, anche lei sorprendente per come contraddice precedenti affermazioni di Eco, è che il comico è strumento di controllo e non può mai diventare critica sociale, funzione che spetterebbe invece all'umorismo per la sua capacità di mettere in discussione i codici culturali:

Humor does not pretend, like carnival, to lead us beyond our own limits. It gives us the feeling, or better, the picture, of the structure of our own limits. It is never off limits, it undermines limits from the inside. It does not fish for impossible freedom, yet it is a true movement of freedom. Humor does not promise us liberation: on the contrary, it warns us about the impossibility of global 
liberation, reminding us of the presence of a law that we have no longer reason to obey. In doing so it undermines the law. (8)

Questa distinzione appare poco soddisfacente, e per una serie di ragioni. Prima di tutto non è chiaro se l'atto della riflessione faccia parte del messaggio o dipenda interamente dal destinatario: ovvero se si possa parlare di opere comiche e lettori umoristi, o se siano le opere stesse a contenere gli elementi della riflessione capaci di 'elevare' il genere a strumento di critica. Nel primo caso, verrebbero meno i presupposti che permettono ad Eco di liquidare la funzione critica del comico carnascialesco, dal momento che nulla impedisce al suo spettatore di elaborarlo in chiave umoristica (e perciò critica). Nel secondo, verrebbero svalutati tutti gli atti comici che non contengano in sé la riflessione, compresa la risata di Franti.

In ogni caso ci troviamo di fronte all'introduzione di una gerarchia di valori che ridimensiona la legittimazione del comico. Di nuovo rispetto al passato c'è solo che gli umoristi hanno guadagnato il diritto ad essere considerati dalla critica ufficiale, anche se solo in base al canone convenzionale che distingue tra chi è capace di descrivere la condizione umana e chi no; ma tutti gli altri, i comici che umoristi non sono, sono di nuovo esclusi. Né si tratta di un gruppo piccolo: restano fuori per lo meno saltimbanchi e buffoni, maschere del carnevale e della commedia dell'arte, personaggi del varietà e del music-hall caro a Joyce, guitti, giullari e clown. A tutti costoro servirebbe di nuovo una giustificazione per sfuggire all'accusa di indulgere in attività fini a se stesse (quando non addirittura funzionali al potere costituito). È proprio la conclusione che Eco sembrava aver respinto nell'Elogio di Franti, e contro cui si schiera apertamente Almansi:

Contro la dittatura del sussiego nella cultura italiana, intendo difendere la libertà di un discorso comico disimpegnato dalla sua funzione sociale. Si tratta di un 
riso che non fustiga i costumi ma gode delle gioie della lingua e del pensiero: un riso disimpegnato, morale solo nel senso che il riso è operazione salutare, benefica (per chi lo suscita e per chi lo subisce), e quindi morale. (Almansi, 1986:10)

Per capire se c'è contraddizione in questo oscillare della posizione di Eco nei confronti del comico, occorre tornare a Il nome della rosa e alla disputa tra Jorge e Guglielmo. Riprenderemo allora dall'ingresso di Jorge nel romanzo, preceduto come abbiamo visto da un brano della regola benedettina contro il riso. Questa teatralissima entrata serve non solo a caratterizzare fin da subito l'estrema severità dello spagnolo, ma anche a introdurre nella discussione la questione delle auctoritates. Quando Guglielmo e Jorge parlano è spesso per citare parole di sommi autori a sostegno delle proprie tesi, e uno dei terreni di scontro fondamentale sarà il tentativo di stabilire se Cristo (ovvero la auctoritas suprema) fosse o meno incline al riso. La questione ha un'importanza strategica fondamentale nella disputa, così come stabilire quale fosse l'effettiva natura della povertà di Cristo è uno dei terreni su cui si giocano le sorti dell'ordine francescano, ovvero il perdurare o meno della sua legittimità.

Le posizioni sono chiare fin dalle prime battute: da una parte la chiusura di Jorge, dall'altra l'apertura di Guglielmo. L'attribuzione dei ruoli suggerisce anche il tipo di strategia retorica che verrà utilizzato dai due: giacché l'intransigente è naturale che attacchi, inveisca, cerchi di proibire; mentre al tollerante non resta che parare $\mathrm{i}$ colpi e smorzare i toni. È Jorge dunque a condurre la discussione, e spetta a lui il ruolo attivo di definizione dei termini del problema. Più che attraverso argomentazioni logiche, la sua requisitoria procede attraverso affermazioni accompagnate dall'uso di formule ieratiche tipiche della preghiera ${ }^{5}$, e da cui emerge un'immagine del comico

\footnotetext{
Si veda ad esempio: "E come ci sono discorsi cattivi ci sono immagini cattive. E sono quelle che mentono circa la forma della creazione e mostrano il mondo al contrario di ciò che deve essere, è sempre stato e sempre sarà nei secoli dei secoli sino alla consunzione dei
} 
sempre più sinistra. In principio esso è strumento di deformazione del reale (ad esempio nei marginalia grotteschi e surreali di Adelmo); quindi un segno di dissoluzione, "cosa assai vicina alla morte e alla corruzione del corpo" (Il nome della rosa, 1980:103); e più avanti ancora, nello scambio di battute che segue, addirittura strumento di negazione della fede:

[Jorge] "L'animo è sereno solo quando contempla la verità e si diletta del bene compiuto, e della verità e del bene non si ride. Ecco perché Cristo non rideva. Il riso è fonte di dubbio."

[Guglielmo] "Ma talora è giusto dubitare."

[Jorge] "Non ne vedo la ragione. Quando si dubita occorre rivolgersi a un'autorità, alle parole di un padre o di un dottore, e cessa ogni dubbio. Così ridendo l'insipiente dice implicitamente 'Deus non est'." (139)

Negando il riso Jorge potrebbe essere spinto anche a negare la necessità di coltivare la teologia, giacché se di questioni di fede si discute è pur sempre perché esistono dubbi sull'interpretazione delle scritture, ma ai padri e ai dottori è lecito discutere e fare chiarezza su questioni legate alla dottrina. Ed è proprio la questione delle auctoritates a ricordarci che nel Medioevo l'unica direzione consentita nelle relazioni sociali e culturali era quella che andava dall'alto (comandi, regole) verso il basso (sottomissione, obbedienza). Il comico invece, in quanto espressione del dubbio, si muove in una direzione contraria permettendo all'individuo ('basso') di mettere in discussione coloro che lo sovrastano nella gerarchia. Legittimato da un'autorità come Aristotele, questo principio critico finirebbe con il diventare un' arma potentissima di emancipazione e riscatto sociale:

Che il riso sia proprio dell'uomo è segno del nostro limite di peccatori. Ma da questo libro quante menti

tempi." (87); e "Gesù non raccontò mai commedie, né favole, ma solo limpide parabole che allegoricamente ci istruiscono su come guadagnarci il Paradiso, e così sia” (137). 
corrotte come la tua trarrebbero l'estremo sillogismo, per cui il riso è il fine dell'uomo! Il riso distoglie, per alcuni istanti, il villano dalla paura. Ma la legge si impone attraverso la paura, il cui nome vero è timor di Dio. E da questo libro potrebbe partire la scintilla luciferina che appiccherebbe al mondo intero un nuovo incendio: e il riso si disegnerebbe come l'arte nuova, ignota persino a Prometeo, per annullare la paura. (477-78)

Il moderato Guglielmo, pur sempre uomo della sua epoca e membro della classe dominante, difficilmente sarebbe arrivato a conclusioni simili, e per questo la sua contro-requisitoria si preoccupa, piuttosto che di difendere la funzione di critica sociale del comico, di attaccare il fanatismo di Jorge: "Tu sei il diavolo [...]. Il diavolo non è il Principe della materia, è l'arroganza dello spirito, la fede senza sorriso, la verità che non viene mai presa dal dubbio" (480-81). L'avversione di Jorge per il riso non trarrebbe dunque origine da un progetto razionale di difesa dell'ordine costituito, ma da un terrore infondato che ha trasformato il bibliotecario in un mostro. Un mostro che merita di essere esporto con brutalità al ridicolo: "Io ti odio, Jorge, e se potessi ti condurrei giù, per il pianoro, nudo con penne di volatili infilate nel buco del culo, e la faccia dipinta come un giocoliere e un buffone, perché tutto il monastero ridesse di te, e non avesse più paura" (481).

In definitiva la posizione di Guglielmo convince poco, e non solo per l'immagine del vecchio cieco denudato, deturpato e trascinato a forza di fronte ai suoi pari, che ben poco ha a che fare con il riso ed il ridicolo. Anche a tragedia consumata, dopo aver riacquistato la sua naturale bonomia, il francescano sintetizza la sua posizione nei confronti del riso in termini che affrontano solo parzialmente le questioni sollevate dall'avversario: "Il compito di chi ama gli uomini è di far ridere della verità, fare ridere la verità, perché l'unica verità è imparare a liberarci dalla passione insana per la verità" (494). Per quanto condivisibile, questa conclusione rievoca le affermazioni un 
po' titubanti dell'introduzione al Diario minimo, che riducevano il campo di applicazione del comico a una singola specifica funzione, e neanche tanto critica. Cosa che spinge a chiedersi: ma se davvero il comico fosse solo un antidoto alla troppa serietà, davvero sarebbe valsa per Jorge la pena di ammazzare tanta gente pur di sopprimerlo?

Si può dire piuttosto che sia proprio Jorge a legittimare il comico, dal momento che gli riconosce una funzione che il nostro sistema di valori spinge a riconoscere come positiva: quella di liberare l'uomo dalla paura e con essa dall'oppressione delle leggi ingiuste. Ma Jorge ci dice anche che questa funzione può essere realizzata solo attraverso l'accettazione del comico da parte dell'élite culturale. Il che equivale a dire che la cultura popolare vera e propria, quella che si esprime indipendentemente dall'establishment, non è in grado da sola di sviluppare strumenti di emancipazione sociale. La famosa legittimazione insomma esiste, ma è subordinata all'attività di quelli che altrove Eco chiama 'operatori culturali' (Apocalittici e integrati, 1965:15, 283), e l'intero svolgimento del tema si può ricondurre alle argomentazioni di Apocalittici e integrati, permettendoci di chiarirne gli aspetti che apparivano a prima vista contraddittori.

In Apocalittici e integrati Eco riconosce il valore di opere della cultura di massa, ma senza sovvertire del tutto la concezione tradizionale di cultura alta o bassa. Ecco allora i frequenti riferimenti ad opere d'arte più e meno valide, secondo canoni che non mettono mai in dubbio, ad esempio, la superiorità in senso assoluto della musica classica rispetto a quella leggera ${ }^{6}$. Il paesaggio culturale dipinto da Eco è ancora fatto di gerarchie, tanto di critici e autori

6 Cfr. Apocalittici e integrati: “[...] L'uomo che fischietta Beethoven perché lo ha sentito alla radio è già un uomo che, sia pure al semplice livello della melodia, ha avvicinato Beethoven (né si può negare che a questo livello si manifesti già in misura semplificata la legalità formale che regge agli altri livelli, armonico, contrappuntistico, ecc. l'opera intera del musicista) mentre un'esperienza del genere un tempo era riservata alle classi abbienti; tra $\mathrm{i}$ cui rappresentanti moltissimi probabilmente, pur sottomettendosi al rituale del concerto, fruivano la musica sinfonica allo stesso livello di superficialità. A questo proposito si citano le cifre impressionanti di musica valida diffusa oggi alla radio o dai dischi, e ci si domanda se questa accumulazione di informazione musicale non debba essersi risolta, in molti casi, in stimolo efficace per acquisizioni culturali autentiche [...]" (46). 
quanto di lettori e spettatori, che possono essere più e meno preparati, di gusto più e meno formato, più e meno colti. Ed è su questa ambiguità di fondo tra l'abbattimento di steccati e gerarchie e la loro conservazione che si gioca l'efficacia delle tesi del libro. Con queste premesse, la risata di Franti non potrà diventare strumento di critica sociale se non nelle pagine di un libro scritto e commentato, ovvero dopo essere passata attraverso un processo di elaborazione culturale svolto da un operatore qualificato. E nemmeno il music-hall sarà mai critica della vita, se non in un distico di James Joyce.

Del resto questo limite è da ritenersi intenzionale, programmatico. Chi volesse considerare la dimensione politica della comicità popolare può sempre rivolgersi a Dario Fo, che in questo senso svolge rispetto ad Eco una funzione complementare. Il nome della rosa potrebbe anzi essere letto insieme a Lu Santu Jullàre Françesco (1999), recuperando una dimensione del racconto che Eco non ci dà. Oltre a mettere in scena la comicità rivoluzionaria di Francesco, Fo ci ricorda anche che la comicità nata dal basso non era poi ritenuta tanto innocua dai potenti ai tempi in cui è ambientato il romanzo, se è vero che appena un secolo prima Federico II aveva promulgato un editto con il quale si "incitava il pubblico a insultare e bastonare i giullari scoperti sulle piazze durante le sagre e i mercati", e che costoro "non godevano del diritto di appellarsi alla giustizia in quanto indegni di entrare nel novero delle persone civili e umane" (Fo, 1999:4).

Eco di questo non parla perché la sua battaglia per l'apertura culturale si svolge su un piano diverso. Non è un movimento che nasce dal basso, ma una critica alla rigidità di parte degli intellettuali che si svolge attraverso i canali consolidati del dibattito tra pari: vale a dire saggi, articoli, conferenze nelle università. Non è una rivoluzione che intende insediare al potere una nuova classe, ma un tentativo di eliminare i pregiudizi verso determinate forme d'arte. Spingersi oltre, legittimare fino in fondo il comico, sarebbe un gesto di diverso significato, tale forse da mettere in dubbio la funzione stessa del critico. 
Le oscillazioni nell'atteggiamento di Eco nei confronti del comico coincidono allora con i limiti imposti alla sua ricerca da un preciso senso delle gerarchie culturali. Ed è proprio questa adesione di fondo al sistema a risultare incompatibile con le conclusioni più radicali della legittimazione, che pure Eco sembra sempre sul punto di trarre, mostrandoci un autore disposto sì a riconoscere il valore di certi prodotti della cultura di massa, ma senza per questo mettere in discussione la funzione, le prerogative e in fin dei conti il primato dell'élite di cui lui stesso fa parte.

\section{Bibliografia}

\begin{tabular}{|c|c|c|}
\hline Almansi, G. & 1986 & La ragion comica. Milan: Feltrinelli. \\
\hline Bakhtin, M. & 1968 & $\begin{array}{l}\text { Rabelais and His World. Cambridge: } \\
\text { M.I.T. Press. }\end{array}$ \\
\hline Bartezzaghi, S. & 1998 & $\begin{array}{l}\text { Sfiga all'OK Corrall. Torino: } \\
\text { Einaudi. }\end{array}$ \\
\hline Baudelaire, C. & 1992 & $\begin{array}{l}\text { Of the Essence of Laughter and } \\
\text { Generally of the Comic in the Plastic } \\
\text { Art. .In Selected Writings on Art and } \\
\text { Artists. Trans Charvet, P.E. } \\
\text { Harmondsworth: Penguin:140-161. }\end{array}$ \\
\hline Bergson, $\mathrm{H}$. & 1911 & $\begin{array}{l}\text { Laughter: an Essay on the Meaning } \\
\text { of the Comic. London: Macmillan. }\end{array}$ \\
\hline Campanile, A. & 1975 & $\begin{array}{l}\text { Se la luna mi porta fortuna. Milano: } \\
\text { Rizzoli. }\end{array}$ \\
\hline Durant, J. e Miller J. & 1988 & $\begin{array}{l}\text { Laughing } \\
\text { Longman. }\end{array}$ \\
\hline Eco, U. & 1965 & $\begin{array}{l}\text { Apocalittici e integrati. Milano: } \\
\text { Bompiani. }\end{array}$ \\
\hline
\end{tabular}




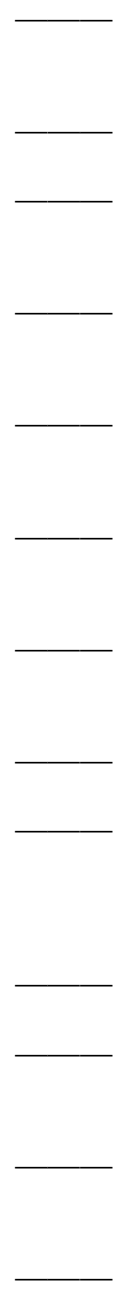

1976 A Theory of Semiotics. Bloomington: Indiana University Press.

1992 Diario minimo. Milano: Bompiani.

1980 Il nome della rosa. Milano: Bompiani.

1988 Il pendolo di Foucault. Milano: Bompiani.

1992 Il secondo diario minimo. Milano: Bompiani.

2000 La bustina di Minerva. Milano: Bompiani.

1962 Le poetiche di Joyce. Milano: Bompiani.

1963 Opera aperta. Milano: Bompiani.

1967 Pirandello ridens. In Sugli specchi e altri saggi. Milano: Bompiani, 1985: 261-270.

1995 Povero Pinocchio. Milano: Comix.

1989 Sei passeggiate nei boschi narrativi. Milano: Bompiani.

1985 Sugli specchi e altri saggi. Milano: Bompiani.

1984 The Frames of Comic 'Freedom'. In Eco U., Ivanov V.V. e Rector M., Carnival! A cura di T. A. Sebeok. Berlino e New York: Mouton Publishers. 


\begin{tabular}{|c|c|c|}
\hline & 1975 & $\begin{array}{l}\text { Trattato di semiotica generale. } \\
\text { Milano: Bompiani. }\end{array}$ \\
\hline & 1973 & $\begin{array}{l}\text { Woody Allen: un everyman per } \\
\text { happy few. In Woody Allen, Saperla } \\
\text { lunga. Milano: Bompiani. }\end{array}$ \\
\hline Ellman , R. & 1959 & $\begin{array}{l}\text { James Joyce. New York: Oxford } \\
\text { University Press. }\end{array}$ \\
\hline Fo, D. & 1999 & $\begin{array}{l}\text { Lu Santu Jullàre Françesco, a cura di } \\
\text { Rame F. Torino: Einaudi. }\end{array}$ \\
\hline Janko, R. & 1984 & $\begin{array}{l}\text { Aristotle on Comedy: Towards a } \\
\text { Reconstruction of Poetics II. London: } \\
\text { Duckworth. }\end{array}$ \\
\hline McFadden, G. & 1982 & $\begin{array}{l}\text { Discovering the Comic. Princeton: } \\
\text { Princeton University Press. }\end{array}$ \\
\hline Queneau, R. & 1983 & $\begin{array}{l}\text { Esercizi di stile. Trad. Umberto Eco. } \\
\text { Torino: Einaudi. }\end{array}$ \\
\hline Zamponi, E. & 1986 & I draghi locopei. Torino: Einaudi. \\
\hline
\end{tabular}

